\title{
Orofacial pain during rest and chewing in people with dementia admitted to acute hospital wards: validity testing of the Orofacial Pain Scale for Non-Verbal Individuals
}

Liza J.M. van de Rijt, DDS

Investigator, Faculty of Dentistry, Department of Oral Kinesiology, Academic Centre of Dentistry Amsterdam (ACTA), University of Amsterdam and Vrije Universiteit Amsterdam, Amsterdam, The Netherlands

Roxane A.F. Weijenberg, PhD

Assistant professor, Faculty of Dentistry, Department of Oral Kinesiology, Academic Centre of Dentistry Amsterdam (ACTA), University of Amsterdam and Vrije Universiteit Amsterdam, Amsterdam, The Netherlands

Alexandra R. Feast, PhD

Research Associate, Marie Curie Palliative Care Research Department, Division of Psychiatry, University College London, London, United Kingdom

Suzanne Delwel, DDS

Investigator, Faculty of Dentistry, Department of Oral Kinesiology, Academic Centre of Dentistry Amsterdam (ACTA), University of Amsterdam and Vrije Universiteit Amsterdam, Amsterdam, The Netherlands and The Department of Clinical Neuropsychology, Faculty of Behavioral and Movement Sciences, Vrije Universiteit Amsterdam, The Netherlands

Victoria Vickerstaff, PhD

Research Statistician, Marie Curie Palliative Care Research Department, Division of Psychiatry, University College London, London, United Kingdom, and The Research Department of Primary Care and Population Health, University College London, London, United Kingdom

Frank Lobbezoo, DDS, PhD

Professor, Chair \& Vice-Dean, Faculty of Dentistry, Department of Oral Kinesiology, Academic Centre of Dentistry Amsterdam (ACTA), University of Amsterdam and Vrije Universiteit Amsterdam, Amsterdam, The Netherlands

Elizabeth L. Sampson, MD, PhD

Reader, Marie Curie Palliative Care Research Department, Division of Psychiatry, University College London, London, United Kingdom and Barnet Enfield and Haringey Mental Health Trust Liaison Psychiatry Team, North Middlesex University Hospital, London, United Kingdom

\section{Corresponding author}

Liza J.M. van de Rijt

Department of Oral Kinesiology

Academic Centre of Dentistry Amsterdam (ACTA)

Room 3N-75

Gustav Mahlerlaan 3004

1081 LA Amsterdam

The Netherlands

1.j.m.vande.rijt@acta.nl 


\begin{abstract}
AIM-The aim of this study was to assess the validity of the components 'resting' and 'chewing' of the recently developed observational diagnostic tool, the Orofacial Pain Scale for Non-Verbal Individuals (OPS-NVI).
\end{abstract}

METHODS-This cross-sectional observational study was carried out in two UK hospitals. Using the OPS-NVI to identify orofacial pain, 56 participants with dementia, admitted to the acute hospital, were observed for 3 minutes during rest and chewing. Thereafter, participants were asked about presence of orofacial pain, using self-report pain scales. The sensitivity, specificity, and Area Under the Receiver Operating Curve (AUROC) of the OPS-NVI were calculated for each activity. The Spearman coefficient was calculated between the number of 'yes'-scored behaviour items of the OPS-NVI and presence of orofacial pain, according to self-report.

RESULTS-Orofacial pain was present in 5.4\% participants during rest, and $9.1 \%$ participants during chewing, using the OPS-NVI. The prevalence of self-reported orofacial pain was $5.4 \%$ during rest, and $10.7 \%$ during chewing. The specificity of the OPS-NVI was $98.1-100 \%$, the sensitivity was $66.7-83.3 \%$, and the AUROC was $0.824-0.917$. The predictive validity shows a strong correlation $(0.633-0.930, \mathrm{p}<0.001)$ between the number of 'yes'-scored behaviour items and the self-reported presence of orofacial pain.

CONCLUSION-The components 'resting' and 'chewing' of the OPS-NVI showed promising concurrent and predictive validity. Nevertheless, further validation is required and highly recommended.

\title{
Keywords
}

Dementia; Orofacial Pain; Facial Pain; Toothache; Hospital; Observation; OPS-NVI; Validation 


\section{INTRODUCTION}

A 2015 report about global ageing from the United Nations shows a substantial recent increase in the number of older people ${ }^{1}$. In 2050 , the population of older people will double in size and this will result in over 100 million people having dementia ${ }^{1,2}$.

Daily, almost half of people with dementia experience pain, which can be difficult to detect, and is therefore likely to be under-treated ${ }^{3-5}$. Undetected pain may lead to distress and cause aggression, depression, agitation, or vocalisations ${ }^{6,7}$. Under-treated pain may increase the risk of delirium, and decreases quality of life $\mathrm{e}^{8,9}$.

Orofacial pain is common in older people, originating from the teeth, the joints and muscles of the masticatory system, or other non-odontogenic tissues ${ }^{10,11}$. Previous studies comparing the prevalence of orofacial pain in people with and without dementia, show a prevalence of $7.4-21.7 \%$ in people with dementia, whereas these studies show a prevalence of $6.7-18.5 \%$ in people without dementia ${ }^{12-14}$.

Adequate diagnosis is essential as a first step in provision of effective treatment. The 'gold standard' for the diagnosis of pain, is self-report ${ }^{15,16}$. For a successful self-report pain assessment, it is important that the person is able to verbally communicate ${ }^{8}$. However, in people with severe dementia, progressive decline of verbal communication may result in inability to answer simple 'yes or no' questions ${ }^{8}$. Therefore, self-report pain scales are not suitable in this population, and direct observation is needed ${ }^{3,8}$.

There is a lack of research and instruments dealing with the assessment of dental and orofacial pain in people with dementia, who are no longer able to communicate verbally ${ }^{11}$. Therefore, the Orofacial-Pain Scale for Non-Verbal Individuals (OPS-NVI) has recently been developed to diagnose orofacial pain in people who are unable to communicate verbally ${ }^{3}$. The OPS-NVI is focused on behaviour items, to explore possible non-verbal communication to 
express orofacial pain. The OPS-NVI consists of four components, namely 'resting', 'chewing', 'drinking', and 'oral hygiene care'

The aim of this study was to assess the concurrent and predictive validity of the 'resting' and 'chewing' components of the OPS-NVI.

\section{MATERIALS AND METHODS}

\section{Design and participants}

All participants in this cross-sectional cohort study were observed during a single assessment in two different hospitals, both in London, UK. Hospital 1 is located in central London, whereas hospital 2 is suburban. Participants were included if they were 70 years or above, had a diagnosis of dementia in their clinical notes, their English language was sufficient to complete the study ratings, and they were able to self-report the presence or absence of pain. Nursing staff identified potential participants, and asked if they could be approached by a researcher. Patients who indicated either verbally, or non-verbally, that they did not wish to participate, were excluded. Patients with delirium, those who were moribund or comatose, or those with clinical concerns that ward nursing staff felt should preclude them being approached, were excluded as well.

\section{Ethics}

The procedure for obtaining informed consent was complied with capacity legislation governing England and Wales (Mental Capacity Act 2005, Sections 30-34). From the participants with the capacity to consent, written informed consent was obtained. If the participant did not have capacity to consent, a personal or professional consultee was asked to follow a structured procedure to give agreement for the person's participation in the study, 
and sign his/her assent for this. The London Queen Square Research Ethical Committee and the UK Health Research Authority reviewed and approved this study (17/LO/0430).

\section{Instruments}

Brief demographic information was collected on age, gender, ethnicity, marital status, number of years in general education, and highest completed level of education. The OPSNVI consists of four components, namely 'resting', 'chewing', 'drinking', and 'oral hygiene care' ${ }^{3}$. For this study, the components 'resting' and 'chewing' were used. During the crosssectional assessment, the participant was observed for 3 minutes during rest, and for 3 minutes during eating a routine meal, or a snack. For each component, i.e., resting and chewing, a score sheet of the OPS-NVI was completed during, or immediately after the observation. Behaviour items of the categories 'facial activities', 'body movements', 'vocalizations', and 'specific' were scored as 'yes', 'no', or 'not applicable'. These items are shown in Table 1. For each activity, the estimated pain intensity was rated with a number between 0 and 10, where 0 is no pain and 10 is pain as bad as it could possibly be. The intensity of the perceived pain was rated by the researcher ${ }^{17}$.

After the observation with the OPS-NVI, the participants were asked if they experienced pain in the orofacial area during each activity. To determine the intensity of orofacial pain, according to self-report, brief self-report pain scales, i.e., the Numeric Rating Scale (NRS), the Verbal Descriptor Scale (VDS), and the Faces Pain Scale Revised (FPS-R), were used, in case pain was present during the activity ${ }^{18-22}$. To determine whether the participant was able to self-report pain, their understanding of the scales was assessed with test-questions. The participants were asked 'Which number reflects more pain; a 3 or a 7?', 'Which word means more pain; moderate or severe?', and 'Which face shows more pain? This one (point to face 2) or this one (point to face 8)?'. If the participants did not answer all test-questions correctly, they were excluded from this study. 


\section{Data analysis}

SPSS Version 24 Software (IBM Corp., Armonk, NY, USA, 2012) was used for data analyses.

\section{Concurrent validity}

Concurrent validity refers to the extent to which the results of a certain test correspond to a previously developed 'gold-standard'. To assess concurrent validity of the OPS-NVI, the estimated pain intensity rated by the researcher was compared with outcomes of the three self-report pain scales, using Spearman's coefficient, with a significance level of $\mathrm{p}<0.05$. This was analysed for the components 'resting' and 'chewing' separately, for both hospitals together. A correlation ( $\mathrm{r}$ ) of 0.5 indicates a large effect, a correlation of 0.3 indicated a medium effect, and a correlation of 0.1 indicated a small effect, according to Cohen's guidelines $^{23}$. The sensitivity, specificity, and the Area Under the Receiver Operating Curve (AUROC) were calculated for each activity by comparing the presence of orofacial pain according to the OPS-NVI, with the presence of pain according to self-report. Orofacial pain, according to the OPS-NVI, was marked as 'present', when the estimated pain intensity was rated greater than, or equal to 1 by the researcher. Orofacial pain, according to the OPS-NVI, was marked as 'absent', when the estimated pain intensity was rated 0 by the researcher. An AUROC of 0.9-1.0 indicates the accuracy of a diagnostic test as 'outstanding', 0.8-0.9 as 'excellent', 0.7-0.8 as 'acceptable', and 0.5 suggests no discrimination ${ }^{24}$.

\section{Predictive validity and agreement}

To determine if the single behaviour items and the total number of 'yes'-scored behaviour items with the OPS-NVI are related to the presence of orofacial pain, according to self-report, the Spearman's coefficient, with a significance level of $\mathrm{p}<0.05$, was used. 
To determine if the presence of orofacial pain according to the OPS-NVI agrees with the presence of orofacial pain, according to self-report, the prevalence-adjusted and bias-adjusted kappa (PABAK) was used ${ }^{25}$.

These were analysed for the activities 'resting' and 'chewing' separately, for both hospitals together. To identify the size of the correlations, Cohen's guidelines were used as well ${ }^{23}$. A PABAK value below 0.4 represents poor agreement, values between 0.4 and 0.75 indicate fair to good agreement and values of 0.75 and higher represent excellent agreement ${ }^{26}$.

\section{RESULTS}

In total, 145 patients were approached by nursing staff. Patients who indicated they did not wish to participate, or consultees who indicated that the patient would not wish to participate, were excluded. If the personal consultee, who gave verbal agreement over the phone, did not return the signed consultee form, the patient did not participate in the study. In 15 cases, patients were discharged from hospital before they could be screened. Informed consent was obtained from 101 patients. However, 45 patients were not able to correctly self-report the presence or absence of pain, i.e., they were not able to answer all test-questions correctly. Therefore, they were excluded from this study, and 56 participants were included. The average age was 84.2 (SD 6.54) years old, and 58.9\% were female. Further demographics are shown in Table 2. There were no significant differences between the two hospitals, concerning the demographics. All 56 participants were observed during rest. One participant received enteral nutrition, which precluded him from being observed during chewing. Therefore, the remaining 55 participants were observed during chewing.

\section{Concurrent validity}

The prevalence of orofacial pain, according to the OPS-NVI, was $5.4 \%$ ( $\mathrm{n}=3$ out of 56 participants) during rest. The prevalence of pain according to self-report was also $5.4 \%$ ( $\mathrm{n}=3$ 
out of 56 participants) during rest. The prevalence of orofacial pain, according to the OPSNVI, was $9.1 \%$ ( $\mathrm{n}=5$ out of 55 participants) during chewing. The prevalence of pain according to self-report in the remaining 55 participants, was $10.7 \%$ ( $\mathrm{n}=6$ out of 55 participants) during chewing. The cross tables with the number of True Positives, True Negatives, False Positives, and False Negatives, are given in Table 3 for each activity separately. The specificity during rest was $98.1 \%$, the sensitivity was $66.7 \%$, and the AUROC was 0.824 , indicating an 'excellent' accuracy. The specificity during chewing was $100 \%$, the sensitivity was $83.3 \%$, and the AUROC was 0.917 , indicating an 'outstanding' accuracy. Since there were only two True Positives during rest, and only five True Positives during chewing, the Spearman correlation between the estimated pain intensity rated by the researcher and self-report pain scales could not be assessed. For each activity, the estimated pain intensity rated by the researcher and the outcomes of the three self-report pain scales, in the participants in whom orofacial pain was present, are given in Table 4.

\section{Predictive validity and agreement}

The correlations between the behaviour items of the OPS-NVI and the presence of orofacial pain according to self-report are shown in Table 5. The correlation between the number of 'yes'-scored behaviour items of the OPS-NVI and presence of orofacial pain according to self-report during rest was $0.633(\mathrm{p}<0.001, \mathrm{n}=56)$, indicating a large effect. The correlation during chewing was $0.930(\mathrm{p}<0.001, \mathrm{n}=55)$, indicating a large effect as well. The PABAK during rest was $92.9 \%$ (95\% C.I. 75.4, 99.1), indicating excellent agreement. The PABAK during chewing was $96.4 \%$ (95\% C.I. 80.6, 99.9), indicating excellent agreement as well.

\section{DISCUSSION}

The aim of this study was to assess the validity of the components 'resting' and 'chewing' of the OPS-NVI. The specificity of the OPS-NVI was 98.1-100.0\%, the sensitivity was 66.7- 
83.3\%, and the AUROC was $0.824-0.917$. The predictive validity shows a strong correlation $(0.633-0.930, \mathrm{p}<0.001)$ between the number of 'yes'-scored behaviour items and the presence of orofacial pain, according to self-report. Furthermore, there was excellent agreement between the presence of orofacial pain according to the OPS-NVI and according to selfreport.

The Spearman correlation between the OPS-NVI and self-report pain scales could not be assessed. This could be explained by the low prevalence of orofacial pain. However, it may also be due to the limited number of people who were able to self-report pain due to the severity of their dementia. The specificity, sensitivity, and AUROC were favourably high. However, it must be considered that only six participants verbally communicated that they were in pain during chewing. It is recommended to further validate the OPS-NVI in a verbal population where the prevalence of pain is higher and where more severe pain is present. Predictive validity shows a strong correlation $(0.633-0.930, \mathrm{p}<0.001)$ between the number of 'yes'-scored behaviour items and presence of orofacial pain, according to self-report. During rest, 'frowning' and 'narrowing or closing eyes' showed a significant strong correlation, and 'opened mouth', 'rubbing', and 'restlessness' showed a significant medium correlation with the presence of self-reported pain. During eating, 'frowning', 'restlessness', 'restricting jaw movement', and 'drooling' showed a significant strong correlation, and 'narrowing or closing eyes' and 'rubbing' showed a significant medium correlation with the presence of selfreported pain. This indicates that participants who self-reported the presence of orofacial pain, where likely to have more of these observed pain indicative behaviour.

\section{Strengths and Limitations}

Although the OPS-NVI was recently developed to identify orofacial pain in non-verbal individuals, it needed further validation. This study is the first one that validated the OPSNVI in an acute hospital setting. 
When verbal communication becomes difficult, or even impossible, observational tools are needed to identify orofacial pain ${ }^{3}$. However, it is important to acknowledge that the observed behaviour could also be caused by other causes of distress, for example pain at other sites of the body or other medical reasons for which the participants were admitted to the hospital ${ }^{27}$. There were only 56 participants in this study who could verbally communicate if they were in pain, and out of this group, only six persons did report pain. We recommend further psychometric evaluation of the OPS-NVI, using a larger sample size and/or a population with a higher prevalence of orofacial pain.

For this study, only the components 'resting' and 'chewing' of the OPS-NVI were used. All participants were admitted to the acute hospital so we could not intervene in their routine daily care on the ward. Therefore, we could not ask them to drink or perform oral care, just for research purposes. However, we were able to observe participants during rest and chewing, since eating food and resting were scheduled parts of their daily routine. All data were collected by one researcher. Therefore, the inter-observer reliability of the OPS-NVI could not be tested. A previous study shows a fair-to-good to excellent interobserver and intra-observer reliability for the component 'chewing ${ }^{28}$. Another recently published study about the psychometric evaluation of the OPS-NVI, indicated that the component 'oral hygiene care' could not be assessed reliably between observers ${ }^{17}$. Furthermore, the components 'drinking' and 'chewing', should be further validated in a population that can communicate verbally and self-report the presence of orofacial pain.

A recently published study indicated that some oral health factors (e.g. brush frequency, indication of chewing quality, consistency of the food, presence of extra-oral abnormalities, person who performed mouth care, and oral hygiene) are significant predictors for the presence of orofacial pain, observed with the OPS-NVI ${ }^{29}$. However, another study examined oral health status in relation to the self-report of orofacial pain, and indicated that oral health 
problems, such as ulcers and caries are frequently present, although no pain was reported ${ }^{17}$. Consequently, the presence of oral health problems, cannot be used as a reference standard for the presence of orofacial pain and oral health examination remains necessary for oral health related quality of life.

\section{Clinical implications}

In the current study, the OPS-NVI was used to identify orofacial pain in people with dementia on acute hospital wards. Adequate diagnosis of orofacial pain is important for providing effective treatment. Since there is no other assessment tool besides the OPS-NVI, to identify orofacial pain in people who are no longer able to communicate verbally, further validation of this observational tool is highly recommended ${ }^{30}$. Until further validation of the OPS-NVI has been performed, it is suggested to use the approach of Herr et al. in clinical situations to identify orofacial pain in people who are no longer able to communicate verbally ${ }^{30,31}$. This approach includes anticipating the presence of possible pain-causing conditions, identifying pain indicators, and establishing a baseline behaviour ${ }^{30,31}$. To clarify whether changes in behaviour are caused by pain, an empirical trial of simple analgesics could be used ${ }^{30,31}$.

\section{Conclusion}

The components 'resting' and 'chewing' of the Orofacial Pain Scale for Non-Verbal Individuals (OPS-NVI) showed promising concurrent and predictive validity. Nevertheless, further validation is required and highly recommended. The components 'drinking' and 'oral hygiene care' of the OPS-NVI also require further validation. It is recommended to further validate the OPS-NVI in a population with a greater prevalence and intensity of orofacial pain, for example in a specialised clinic for dental care for older people. 


\section{Acknowledgements}

The authors would like to thank Ioana Tofan, Dr. Catherine Bond, and all the ward staff, for their contribution to the recruitment of participants. We appreciate the assistance of our professional consultee Dr. Ada Chime. We would like to thank Dr. M. Smalbrugge, for reviewing the study protocol.

Author contributions: L.J.M. van de Rijt, E.L. Sampson, S. Delwel, and F. Lobbezoo conceived and designed the study. A.R. Feast was involved in additional study planning and implementation. V.

Vickerstaff, L.J.M. van de Rijt, R.A.F. Weijenberg, S. Delwel, and A.R. Feast were involved in the processing, analysis and the interpretation of data. L.J.M. van de Rijt drafted the manuscript. All authors were involved in revising the manuscript critically and gave final approval of the manuscript. E.L. Sampson and V. Vickerstaff are supported by Marie Curie core grant funding to the Marie Curie Palliative Care Research Department at University College London, grant MCCC-FCO-16-U.

\section{Conflict of Interest}

The authors declare that the research was conducted in the absence of conflicts of interest.

\section{References}

1. United Nations DoEaSA, Population Division. World Population Ageing 2015. (ST/ESA/SERA/390). 2015.

2. International AsD. The Global Impact of Dementia 2013-2050. Policy Brief for Heads of Government. 2013.

3. van Kooten J, Delwel S, Binnekade TT, et al. Pain in dementia: prevalence and associated factors: protocol of a multidisciplinary study. BMC geriatrics. 2015;15:29.

4. Horgas AL, Tsai PF. Analgesic drug prescription and use in cognitively impaired nursing home residents. Nursing research. 1998;47(4):235-242.

5. Sengstaken EA, King SA. The problems of pain and its detection among geriatric nursing home residents. Journal of the American Geriatrics Society. 1993;41(5):541-544.

6. Ryden MB, Feldt KS, Oh HL, et al. Relationships between aggressive behavior in cognitively impaired nursing home residents and use of restraints, psychoactive drugs, and secured units. Archives of psychiatric nursing. 1999;13(4):170-178.

7. Sampson EL, White N, Lord K, et al. Pain, agitation, and behavioural problems in people with dementia admitted to general hospital wards: a longitudinal cohort study. Pain. 2015;156(4):675-683.

8. Zwakhalen SM, Hamers JP, Abu-Saad HH, Berger MP. Pain in elderly people with severe dementia: a systematic review of behavioural pain assessment tools. BMC geriatrics. 2006;6:3.

9. Inouye SK. Delirium in hospitalized elderly patients: recognition, evaluation, and management. Connecticut medicine. 1993;57(5):309-315.

10. Renton T, Durham J, Aggarwal VR. The classification and differential diagnosis of orofacial pain. Expert review of neurotherapeutics. 2012;12(5):569-576. 
11. Lobbezoo F, Weijenberg RA, Scherder EJ. Topical review: orofacial pain in dementia patients. A diagnostic challenge. Journal of orofacial pain. 2011;25(1):6-14.

12. de Souza Rolim T, Fabri GM, Nitrini R, et al. Oral infections and orofacial pain in Alzheimer's disease: a case-control study. Journal of Alzheimer's disease : JAD. 2014;38(4):823-829.

13. Adam H, Preston AJ. The oral health of individuals with dementia in nursing homes. Gerodontology. 2006;23(2):99-105.

14. Delwel S, Binnekade TT, Perez RS, Hertogh CM, Scherder EJ, Lobbezoo F. Oral health and orofacial pain in older people with dementia: a systematic review with focus on dental hard tissues. Clinical oral investigations. 2016.

15. Stahnisch FW. Objectifying "Pain" in the Modern Neurosciences: A Historical Account of the Visualization Technologies Used in the Development of an "Algesiogenic Pathology", 1850 to 2000. Brain sciences. 2015;5(4):521-545.

16. Gregory J. The complexity of pain assessment in older people. Nursing older people. 2015;27(8):16-21.

17. Delwel S, Perez R, Maier AB, et al. Psychometric evaluation of the Orofacial Pain Scale for Non-Verbal Individuals as a screening tool for orofacial pain in people with dementia. Gerodontology. 2018.

18. Hartrick CT, Kovan JP, Shapiro S. The numeric rating scale for clinical pain measurement: a ratio measure? Pain practice : the official journal of World Institute of Pain. 2003;3(4):310316.

19. Krebs EE, Carey TS, Weinberger M. Accuracy of the pain numeric rating scale as a screening test in primary care. Journal of general internal medicine. 2007;22(10):1453-1458.

20. Herr KA, Spratt K, Mobily PR, Richardson G. Pain intensity assessment in older adults: use of experimental pain to compare psychometric properties and usability of selected pain scales with younger adults. The Clinical journal of pain. 2004;20(4):207-219.

21. Hicks CL, von Baeyer CL, Spafford PA, van Korlaar I, Goodenough B. The Faces Pain Scale-Revised: toward a common metric in pediatric pain measurement. Pain. 2001;93(2):173-183.

22. Williamson A, Hoggart B. Pain: a review of three commonly used pain rating scales. Journal of clinical nursing. 2005;14(7):798-804.

23. Cohen J. Statistical Power Analysis for the Behavioral Sciences. Routledge; 1988.

24. Wiley J, and Sons. Applied Logistic Regression. Vol 2nd Ed. Chapter 5. New York2000.

25. Chen G, Faris P, Hemmelgarn B, Walker RL, Quan H. Measuring agreement of administrative data with chart data using prevalence unadjusted and adjusted kappa. BMC medical research methodology. 2009;9:5.

26. Landis JR, Koch GG. The measurement of observer agreement for categorical data. Biometrics. 1977;33(1):159-174.

27. Jordan A, Regnard C, O'Brien JT, Hughes JC. Pain and distress in advanced dementia: choosing the right tools for the job. Palliative medicine. 2012;26(7):873-878.

28. de Vries MW, Visscher C, Delwel S, et al. Orofacial Pain during Mastication in People with Dementia: Reliability Testing of the Orofacial Pain Scale for Non-Verbal Individuals. Behavioural neurology. 2016;2016:3123402.

29. van de Rijt LJM, Weijenberg RAF, Feast AR, Vickerstaff V, Lobbezoo F, Sampson EL. Oral health and orofacial pain in people with dementia admitted to acute hospital wards: observational cohort study. BMC geriatrics. 2018;18(1):121.

30. Lobbezoo F, Delwel S, Weijenberg RAF, Scherder EJA. Orofacial Pain and Mastication in Dementia. Current Alzheimer research. 2017;14(5):506-511.

31. Herr K, Bjoro K, Decker S. Tools for assessment of pain in nonverbal older adults with dementia: a state-of-the-science review. Journal of pain and symptom management. 2006;31(2):170-192. 
Table 1: Behaviour items of the OPS-NVI

\begin{tabular}{|c|c|c|}
\hline Category & Behaviour & Meaning of items \\
\hline \multirow[t]{5}{*}{ Facial activities } & Frowning & Lowering and drawing brows together \\
\hline & Narrowing or closing eyes & $\begin{array}{l}\text { Narrowed eyes with tension around the eyes; } \\
\text { not just blinking }\end{array}$ \\
\hline & Raising upper lip & Upper lip raised, nose may be wrinkled \\
\hline & Opened mouth & The lips are parted, jaw is dropped \\
\hline & Tightened lips & $\begin{array}{l}\text { Lips are pressed together and appear more } \\
\text { narrow }\end{array}$ \\
\hline \multirow[t]{4}{*}{ Body movements } & Resisting care & Resisting care, being uncooperative \\
\hline & Guarding & $\begin{array}{l}\text { Protecting affected area, holding body part, } \\
\text { avoiding touch, moving away }\end{array}$ \\
\hline & Rubbing & Tugging or massaging affected area \\
\hline & Restlessness & $\begin{array}{l}\text { Fidgeting, wringing hands, rocking back and } \\
\text { forth }\end{array}$ \\
\hline \multirow[t]{4}{*}{ Vocalizations } & Using offensive words & Cursing, swearing, or using foul language \\
\hline & Using pain-related words & $\begin{array}{l}\text { Using pain words, like "ouch", "ow", or } \\
\text { "that hurts" }\end{array}$ \\
\hline & Screaming/shouting & Using a loud voice to express sounds/words \\
\hline & Groaning & Making a deep, inarticulate sound \\
\hline \multirow[t]{3}{*}{ Specific } & Restricting jaw movement & Making smaller jaw movements than possible \\
\hline & Refusing prosthetics & Removing prosthetics again and again \\
\hline & Drooling & Flowing of saliva outside the mouth \\
\hline
\end{tabular}


Table 2: Descriptive analysis of demographic characteristics of all participants and of each hospital separately

\begin{tabular}{|c|c|c|c|}
\hline & Total $(n=56)$ & Hospital $1(n=14)$ & Hospital $2(n=42)$ \\
\hline \multicolumn{4}{|l|}{ Gender $[\mathrm{n}(\%)]$} \\
\hline Female & $33(58.9)$ & $5(35.7)$ & $28(66.7)$ \\
\hline Male & $23(41.1)$ & $9(64.3)$ & $14(33.3)$ \\
\hline Age $M, S D$ (range) & $84.2,6.5(70-97)$ & $82.0,7.0(70-92)$ & $84.9,6.3(73-97)$ \\
\hline \multicolumn{4}{|l|}{ Ethnicity [n (\%)] } \\
\hline White & $40(71.4)$ & $12(85.7)$ & $28(66.7)$ \\
\hline Mixed/Multiple ethnic groups & $0(0)$ & $0(0)$ & $0(0)$ \\
\hline Asian/Asian British & $6(10.7)$ & $1(7.1)$ & $5(11.9)$ \\
\hline Black/African/Caribbean/Black British & $6(10.7)$ & $1(7.1)$ & $5(11.9)$ \\
\hline Other ethnic group & $4(7.1)$ & $0(0)$ & $4(9.5)$ \\
\hline \multicolumn{4}{|l|}{ Marital Status [n (\%)] } \\
\hline Married & $19(33.9)$ & $4(28.6)$ & $15(35.7)$ \\
\hline Divorced & $6(10.7)$ & $3(21.4)$ & $3(7.1)$ \\
\hline Widowed & $19(33.9)$ & $2(14.3)$ & $17(40.5)$ \\
\hline Single & $12(21.4)$ & $5(35.7)$ & $7(16.7)$ \\
\hline Years in general education $M, S D$ (range) & $10.7,3.1(6-18)$ & $10.8,2.8(7-18)$ & $10.6,3.2(6-18)$ \\
\hline \multicolumn{4}{|l|}{ Highest completed level of education [n (\%)] } \\
\hline Higher degree & $0(0)$ & $0(0)$ & $0(0)$ \\
\hline Degree & $2(3.6)$ & $1(7.1)$ & $1(2.4)$ \\
\hline A level (or equivalent) & $2(3.6)$ & $0(0)$ & $2(4.8)$ \\
\hline HNC/HND (or equivalent) & $0(0)$ & $0(0)$ & $0(0)$ \\
\hline NVQ (or equivalent) & $0(0)$ & $0(0)$ & $0(0)$ \\
\hline GCSE (or equivalent) & $5(8.9)$ & $2(14.3)$ & $3(7.1)$ \\
\hline No qualification & $47(83.9)$ & $11(78.6)$ & $36(85.7)$ \\
\hline
\end{tabular}


Table 3: Cross table of the presence of orofacial pain, observed by the researcher, using the OPS-NVI, with the presence of orofacial pain, according to self-report, during rest and chewing separately

\begin{tabular}{|c|c|c|c|c|c|c|c|c|}
\hline \multirow[t]{3}{*}{ Resting } & \multicolumn{8}{|c|}{ Chewing } \\
\hline & \multicolumn{3}{|c|}{ Self-report } & \multicolumn{5}{|c|}{ Self-report } \\
\hline & & Yes & No & Total & & Yes & No & Total \\
\hline \multirow[t]{2}{*}{ OPS-NVI } & Yes & TP 2 & FP 1 & 3 & OPS-NVI & TP 5 & FP 0 & 5 \\
\hline & No & FN 1 & TN 52 & 53 & & FN 1 & TN 49 & 50 \\
\hline
\end{tabular}

Note. $O P S-N V I=$ Orofacial-Pain Scale for Non-Verbal Individuals, $T P=$ True Positive, $T N=$ True Negative, FP = False Positive, $F N=$ False Negative.

Table 4: The estimated pain intensity rated by the researcher, using the OPS-NVI and the outcomes of the self-report pain scales in all participants who self-reported the presence of orofacial pain, during rest and/or chewing

\begin{tabular}{lllllllll}
\hline & Resting & \multicolumn{5}{l}{ Chewing } \\
\hline & $\begin{array}{l}\text { Obser- } \\
\text { vation }\end{array}$ & Self-report & & $\begin{array}{l}\text { Obser- } \\
\text { vation }\end{array}$ & \multicolumn{2}{l}{ Self-report } \\
\hline Participant & OPS-NVI & NRS & VDS & FPS-R & OPS-NVI & NRS & VDS & FPS-R \\
\hline $\mathbf{1}$ & 2 & 6 & Severe & 4 & 3 & 6 & Severe & 4 \\
\hline $\mathbf{2}$ & 0 & 5 & Moderate & 4 & 0 & 6 & Moderate & 6 \\
\hline $\mathbf{3}$ & 3 & 4 & Moderate & 6 & 4 & 6 & Severe & 8 \\
\hline $\mathbf{4}$ & 2 & 0 & None & 0 & 3 & 2 & Mild & 4 \\
\hline $\mathbf{5}$ & 0 & 0 & None & 0 & 2 & 5 & Moderate & 6 \\
\hline $\mathbf{6}$ & 0 & 0 & None & 0 & 4 & 3 & Mild & 2 \\
\hline
\end{tabular}

Note. $O P S-N V I=$ Orofacial-Pain Scale for Non-Verbal Individuals, $N R S=$ Numeric Rating Scale, VDS = Verbal Descriptor Scale, FPS-R = Faces Pain Scale Revised. 
Table 5: The correlations between the behaviour items of the OPS-NVI and the presence of orofacial pain according to self-report

\begin{tabular}{lllll}
\hline Behaviour & Resting & & Eating & \\
\hline & r & p-value & r & p-value \\
\hline Frowning & 0.648 & $<0.001$ & 0.813 & $<0.001$ \\
Narrowing or closing eyes & 0.567 & $<0.001$ & 0.389 & \\
Raising upper lip & -0.032 & 0.814 & - & 0.003 \\
Opened mouth & 0.296 & 0.027 & - & - \\
Tightened lips & - & - & - & - \\
& & & & - \\
\hline Resisting care & - & - & - & - \\
Guarding & - & - & - & - \\
Rubbing & 0.382 & 0.004 & 0.389 & 0.003 \\
Restlessness & 0.382 & 0.004 & 0.555 & $<0.001$ \\
\hline Using offensive words & - & - & - & - \\
Using pain-related words & - & - & - & - \\
Screaming/shouting & - & - & - & - \\
Groaning & - & - & - & - \\
\hline Restricting jaw movement & - & - & 0.686 & $<0.001$ \\
Refusing prosthetics & - & - & - & \\
Drooling & - & - & 0.555 & - \\
& & & & $<0.001$ \\
\hline
\end{tabular}

Note. OPS-NVI = Orofacial-Pain Scale for Non-Verbal Individuals, $r=$ correlation . 